\title{
L'espace ambigu des disparitions politiques
}

\section{Daniel Hermant}

\section{OpenEdition \\ 12 Journals}

\section{Édition électronique}

URL : http://journals.openedition.org/conflits/189

DOI : $10.4000 /$ conflits.189

ISSN : 1777-5345

Éditeur :

CCLS - Centre d'études sur les conflits lilberté et sécurité, L'Harmattan

\section{Édition imprimée}

Date de publication : 15 mai 1994

ISSN : 1157-996X

\section{Référence électronique}

Daniel Hermant, «L'espace ambigu des disparitions politiques », Cultures \& Conflits [En ligne], 13-14 | printemps-été 1994, mis en ligne le 14 mars 2006, consulté le 30 mars 2021. URL : http:// journals.openedition.org/conflits/189; DOI : https://doi.org/10.4000/conflits.189

Ce document a été généré automatiquement le 30 mars 2021.

Creative Commons License 


\title{
L'espace ambigu des disparitions politiques
}

\author{
Daniel Hermant
}

1 Les disparitions sont une pratique greffée en amont sur des actes de coercition et en aval sur les prisons, les camps et la mort, quelquefois la libération. Elles renvoient à des cas individuels, à des séries ou à un phénomène de masse. Réfléchir sur les disparitions c'est revenir, à travers l'examen des politiques coercitives menées dans une bonne trentaine de pays, sur les rapports de violence qui lient gouvernants et gouvernés en scrutant l'exercice du pouvoir dans ce qu'il a de plus fondamental : le contrôle de la population. Cette approche des politiques de coercition à partir du corpus réuni patiemment par les défenseurs des droits de l'homme, nous oblige "à tailler large" car il est impossible de tracer sans arbitraire sur des critères de simple description une limite significative entre disparitions, exécutions extrajudiciaires, assassinats politiques, massacres, déportations de population. Cet article a pour objectif de recadrer la réflexion sur les disparitions en substituant aux grilles de lecture éthiques ou juridiques couramment utilisées, une grille de lecture sociologique qui seule, à notre sens, permet de réfléchir sur l'unité de la notion. C'est dire que nous scruterons en priorité le pouvoir, ou les masques derrière lesquels il se cache, et non, comme le fait légitimement la littérature sur les violations des droits de l'homme, les victimes sur qui porte la violence ${ }^{1}$. Contraindre : les agencements coercitifs ${ }^{2}$ Les politiques coercitives peuvent dans leurs grandes lignes être analysées à l'aide de deux grandes figures. La première s'organise autour d'une logique d'hostilité absolue dans laquelle le pouvoir cherche à exterminer un adversaire considéré comme un véritable ennemi, et non à le rallier ou à le convaincre. C'est ce type de relation ami/ennemi que Carl Schmit présente comme constitutive du politique mais qui l'est surtout de la guerre: il fonctionne sur la base de la polarisation duelle et de l'absence de Tiers ${ }^{3}$. La seconde correspond à une logique de quadrillage dont l'objet n'est pas d'éliminer une population, mais de disperser une opposition politique afin de la rendre inoffensive. Il s'agit d'une relation de violence portant sur les "choses" ou sur les "corps" qui vise à atomiser la société en empêchant l'émergence d'un authentique espace politique polarisé autour d'enjeux et d'acteurs clairement identifiés. Le dispositif répressif mis en 
place vise à briser les solidarités entre individus ou groupes sans éliminations massives. Progressivement, en quelque sorte par capillarité, la peur provoquée par la répression discrète serions-nous tentés de dire ${ }^{4}$ - de militants d'organisations clairement identifiées, ou quelquefois d'anonymes, est instillée à leurs proches, puis à des franges plus larges de la population ce qui a pour effet d'empêcher la mobilisation de groupe et de freiner l'action collective. C'est dire que cette relation de violence est inséparable d'une relation de pouvoir qui opère au niveau politique en structurant - il vaudrait mieux dire en destructurant - le champ d'action de l'opposition. Il s'agit bien d'une action sur une action pour reprendre l'expression par laquelle Michel Foucault désignait la nature même du pouvoir politique ${ }^{5}$. Ces deux principes d'intelligibilité qui serviront de support à l'analyse, ne doivent pas être interprétés comme délimitant des ensembles séparés, mais plutôt comme la double polarité autour de laquelle se disposent les politiques répressives. Les disparitions, assassinats et massacres qui correspondent à notre première figure, se rencontrent d'abord dans les zones de guerre où s'opposent gouvernements et mouvements armés. L'exemple paradigmatique pourrait en être les massacres perpétrés par les autorités irakiennes au Nord contre les Kurdes et au Sud contre les Chiites des marais, où se mêlent d'une manière complexe la situation de guerre et la répression brutale ${ }^{6}$. Ces violences contre des populations civiles et des individus désarmés n'ont que peu à voir avec l'échange de coups qui lie deux adversaires dans la relation guerrière telle que la définit Clausewitz ${ }^{7}$ - pour les militaires un massacre, ne l'oublions pas, ne doit pas être confondu avec un combat -, et pourtant le fait qu'elles accompagnent ou prolongent des événements proprement militaires, qu'elles concernent en priorité les populations vivant dans les zones de combat, et que leur finalité meurtrière ne se distingue en rien de celle de la guerre, favorise la confusion: guerre donc puisque la finalité reste celle de la guerre, l'élimination physique de l'adversaire, mais guerre civile, au sens de guerre contre les civils, c'est à dire d'extermination de ceux qui sont dépourvus de moyens de défense. Ce mélange entre la violence guerrière et la violence péri-guerrière de la répression, est encore renforcé dans la conscience des exécutants par un discours explicatif commun dont l'objet est de stigmatiser l'ennemi et de légitimer la guerre qui seule pourra l'extirper. Ce discours du pouvoir s'applique à tous et renvoie aussi bien aux opérations proprement militaires qu'aux exactions contre la population locale. Il peut par extension être appliqué à l'ensemble du pays. Si l'action répressive et l'action guerrière doivent être soigneusement séparées par l'analyse, il se trouve que pour les protagonistes locaux engagés dans un "combat" la distinction est soigneusement gommée par le transfert et la globalisation de la rhétorique militaire ami/ennemi à l'ensemble des relations politiques gouvernants/gouvernés. Une seconde difficulté de vocabulaire doit être levée. Considérer une partie de la population d'un pays comme un adversaire intérieur, i.e. comme un corps étranger qu'il faut éradiquer à tout prix, nourrit des logiques d'extermination larges, surtout quand la monopolisation des ressources militaires et communicationnelles par le gouvernement lui donne une grande marge de manoeuvre à l'égard de l'opinion internationale, nous y reviendrons. Aussi l'expression de génocide apparaît-elle souvent dans la littérature sur le tiers monde pour caractériser ces situations ${ }^{8}$. Le paradigme de ces logiques est l'holocauste des juifs pendant la deuxième guerre mondiale 9 . Or il s'agit là d'un phénomène autocentré dont la finalité redouble la nature, ce qui lui confère une dimension "métapolitique" et empêche d'en faire un simple élément violent pouvant s'inscrire dans un projet politique plus vaste. Au contraire, dans les cas qui nous intéressent, les 
exactions aussi sanglantes soient-elles peuvent - et doivent - être resituées dans le cadre plus large d'une gestion politique du conflit. Le massacre qui n'y est jamais une fin en soi est d'ailleurs souvent tempéré par des politiques "démographiques": possibilité de fuite -quelquefois massive - à l'extérieur, dispersion des populations rétives à l'intérieur du pays, et inversement politique de "transmigration" qui installe de nouvelles populations dans les zones à contrôler ${ }^{10}$. L'élimination physique des populations étant subordonnée à des objectifs politiques, réversibles par nature, et le rapport idéologique à l'extermination totale des membres d'un groupe persécuté n'étant pas spécifié, nous n'utiliserons pas le terme génocide qui nous paraît source de confusion dans la description et l'explication à la fois des guerres et des politiques coercitives. L'expression politicide est probablement plus adaptée ${ }^{11}$. A côté de la figure de la guerre que la définition schmittienne étend au politique, nous avions distingué la figure générale du contrôle de population. Or contrairement à ce qui se passe dans les pays occidentaux où la violence dans les rapports de pouvoir est dissimulée, ou légitimée par la notion wéberienne de violence légitime ${ }^{12}$, dans les sociétés qui nous intéressent la violence fait partie du contrat politique ordinaire et le massacre de populations peut précéder des retournements d'alliance surprenant pour un occidental. Les politiques coercitives que nous tentons de cerner renvoient donc à un style de rapports politiques où la violence est un moyen licite de gérer au vu et au su de tous les rapports de certains groupes avec l'Etat. Nous avions pour analyser le champ des politiques coercitives imaginé qu'il était structuré par deux types de relations : les relations de violence portant sur les "choses " ou les "corps" - dont la forme la plus accomplie est la guerre -, et les relations de pouvoir - politique si l'on veut - qui ne fonctionnent que parce que l'autre est "reconnu et maintenu jusqu'au bout comme sujet d'action, et que s'ouvre(...)tout un champ de réponses, réactions, effets, inventions possibles"13, dans un autre vocabulaire, une dynamique d'interaction. Opposer ces deux figures est exagéré, le même événement pouvant s'inscrire simultanément dans les deux registres selon l'échelle où il est examiné. Les acteurs militaires ou paramilitaires se placent, sur le terrain, en position de lutte contre un ennemi intérieur qu'ils cherchent à exterminer physiquement, mais parallèlement au niveau du gouvernement, les acteurs politiques peuvent à travers des transactions resituer les violences à l'intérieur d'une logique politique de contrôle de la population. Y voir une double polarisation, comme nous l'avons écrit ci-dessus, est plus juste que de les opposer, mais encore réducteur. En fait il s'agit d'un rapport métonymique complexe qu'il faut déchiffrer à chaque fois.

2 Faire disparaître Nous avons jusqu'à présent examiné le cadre théorique qui permet d'articuler les politiques coercitives, la figure de la guerre, et celle du pouvoir, mais nous n'avons pas encore caractérisé leur lien avec les disparitions. Pour le faire il faut à la fois préciser les acteurs et l'échelle de l'observation. Arbitre principal de la coercition, le gouvernement structure en amont la scène de la violence et fabrique le cadre général dans lequel va se dérouler la répression. Le discours idéologique contre l'ennemi intérieur qu'il prononce peut rester métaphorique, c'est à dire rester sur le registre politique de l'influence, ou au contraire être pris au pied de la lettre et se traduire par des comportements meurtriers. Mais le gouvernement n'agit qu'à travers des relais et sa maîtrise de la coercition est bien souvent duopolistique plus que monopolistique en cas de guerre civile dans les régions de combats. Aussi il nous faut examiner avec attention le rôle et les objectifs des acteurs répressifs directs : appareils policiers et militaires à la disposition des autorités ${ }^{14}$, mais également organisations de 
lutte armée, tant il est vrai que les disparitions ne sont pas le monopole du pouvoir en place. Ce rôle n'a pas encore fait l'objet d'études académiques. Il est cependant de mieux en mieux connu à travers le matériau empirique que la "littérature humanitaire de dénonciation et d'enquête" sur les violations des droits de l'homme publiée par les instances internationales ou les ONG ${ }^{15}$. Pourquoi? Dans les guerres civiles ${ }^{16}$, les disparitions sont provoquées par le désir des forces armées d'établir par la terreur leur contrôle sur une population soupçonnée de connivence ou de collusion avec la guérilla. Elles prolongent à l'égard de la population locale, mais sous une autre forme, les opérations militaires contre les mouvements armés, et sont inséparables d'autres violences collectives: intimidations, déplacements de population, assassinats, massacres..."Les organes de sécurité et de défense de l'Etat sont entraînés pour poursuivre un ennemi collectif et considèrent en général que les victimes en font partie. Dans bon nombre de cas, ils appliquent le principe qui a fait recette pendant la guerre au Salvador et qui consiste à établir une relation directe entre par exemple un syndicat ou des organisations paysannes et les forces de la subversion. Lorsqu'ils mènent des actions anti-insurrectionnelles, ils ne considèrent pas ces sujets passifs comme des victimes "indépendantes" mais comme des éléments faisant partie de

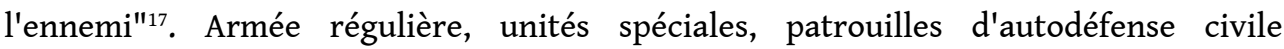
organisées sous contrôle du commandement militaire, unités paramilitaires clandestines, milices privées sont responsables du climat de terreur et des disparitions. En face les organisations armées comme le Sentier Lumineux, le LTTE, le JVP, le NPA ${ }^{18}$, structurées pour contrôler un territoire utilisent à peu près les mêmes méthodes à l'égard de la population. Si les disparitions constituent vraisemblablement une part plus faible dans leur panoplie de violence, c'est parce que les mouvements armés contrôlent des zones plus étroites et pratiquent plus volontiers l'assassinat ou la prise d'otage contre rançon ${ }^{19}$. Les disparitions sont aussi un moyen de voiler l'importance de la répression, de cacher les rafles dans des villages ou des camps, ou de minimiser une politique plus systématique de destructions de villages et de communautés aux yeux de l'opinion interne dont il ne faut pas troubler la quiétude, ou à l'égard de la communauté internationale. Elles sont le maillon central d'une véritable politique de désinformation qui nie la responsabilité de l'armée dans la disparition de paysans pourtant arrêtés par des militaires au vu et su de tout le monde, qui impute la responsabilité des massacres de l'armée à la guérilla, ou qui utilise des groupes paramilitaires clandestins dont tout le monde sait - sans forcément pouvoir le prouver que se sont de véritables organisations-écran constituées de toutes pièces par l'armée. Dans certains cas une véritable dramaturgie de la terreur est mise en place à travers des groupes aux noms mystérieux qui avant d'enlever et d'assassiner doivent terroriser ${ }^{20}$. D'une manière plus générale la multiplication dans les pays du TiersMonde des groupes paramilitaires d'autodéfense soutenus et équipés par l'armée pendant les années 80 s'est traduite par une augmentation sensible des disparitions et des assassinats politiques ${ }^{21}$. Cette volonté de secret se prolonge assez logiquement par des enlèvements de témoins, ou d'enquêteurs membres d'organisation de défense des droits de l'homme pour empêcher l'information de circuler. La recherche du renseignement pour éliminer les cadres des organisations ou des partis clandestins est la troisième origine des disparitions. Des arrestations sont effectuées par des unités du renseignement militaire ou par des groupes paramilitaires, à des fins d'interrogatoire et d'élimination. Ces pratiques très fréquentes dans les régions de combat sont là aussi accompagnées par des pressions sur les familles ou l'entourage des militants enlevés. 
Dans les grandes villes où il n'y a pas de combat à proprement parler, la stratégie de démantèlement des organisations d'opposition prend le profil plus traditionnel de la lutte contre des réseaux clandestins, menée par la police politique ou les unités de renseignement de l'armée, qui est également prolongée par une politique d'intimidation sur l'entourage et les familles. Ces objectifs produisent des effets. Le premier, nous venons de le voir, vise à augmenter par l'intermédiaire du secret, l'efficacité de la répression en dissimulant l'affrontement aux yeux du plus grand nombre, ce qui élargit la marge de manoeuvre des autorités ou de leurs agents. Le second augmente la cruauté de la répression d'une peine supplémentaire, car un enlèvement ne signifiant pas toujours la mort, l'incertitude qui l'accompagne empêche l'indispensable travail de deuil. L'emprisonnement au long cours produit un troisième effet, non plus d'aggravation mais de brouillage. En faisant disparaitre d'une main mais en se réservant la possibilité de libérer de l'autre, le pouvoir ouvre un espace de complicité/chantage avec l'entourage de la victime, voire avec les organisations de défense des droits de l'homme; chacun sait que travailler efficacement pour les victimes se fait en silence ${ }^{22}$ ! Enfin ultime effet de brouillage, en dérobant la violence aux regards du plus grand nombre, les disparitions escamotent l'affrontement direct, voilent le conflit et empêchent les mobilisations collectives. Par qui ? Définie à travers ses objectifs et ses effets généraux, la politique de disparition s'inscrit dans le cadre de la lutte du gouvernement contre la subversion dont nous parlions plus haut. En Amérique latine, ce combat a pris une allure de mouvement international structuré autour d'une idéologie scellée par l'anticommuniste ${ }^{23}$, que complétait un échange très important ${ }^{24}$ de technologies policières contre-insurrectionnelles. Ailleurs il emprunte des voies plus traditionnelles faisant référence à l'identité culturelle ou communautaire, mais quelles que soient les formes du discours politique qui soutiennent les disparitions, les forces de l'ordre qui y sont impliquées entretiennent un rapport privilégié avec l'appareil d'Etat et tout spécialement avec son sommet, ce qui ne surprendra pas dans des régimes de pouvoir personnel. Au Maroc, le roi fut longtemps considéré comme le grand ordonnateur de la politique de disparitions dont les instruments étaient la Garde royale, la Gendarmerie, la Direction de la surveillance du territoire (D.S.T.), les Renseignements généraux, la Direction générale des études et documentation. Au Chili, la D.I.N.A. (direction de l'intelligence nationale) créée par le colonel Contreras, entretenait un rapport direct avec le général Pinochet. Au Tchad, la commission d'enquête instituée après la chute d'Hissène Habré a mis en évidence les responsabilité de la sécurité présidentielle (S.P.) de la Direction de la documentation et de la sécurité (D.D.S.), du service d'investigation présidentielle (S.I.P.) et des renseignements généraux(R.G.) ${ }^{25}$. Cette greffe au plus haut niveau de l'Etat, assure aux agents une autonomie forte, doublée par un sentiment d'impunité puisque bien souvent la répression s'effectue dans le cadre de l'Etat d'urgence qui donne une vaste marge de manoeuvre: "La loi martiale n'étant ni plus ni moins que la volonté du général qui commande l'armée" ${ }^{26}$. Cette sociologie en pointillés des forces de sécurité semble nous inviter à opposer des appareils policiers ou militaires modernes (Argentine, Chili), fonctionnant plus ou moins en vase clos, mais capables de sélectionner finement les victimes pour instiller une politique de terreur, à des instruments de répression plus grossiers voués au massacre sur le terrain (Guatemala, Sri Lanka, Tchad...) pour lesquels les disparitions constitueraient un moyen de dissimuler les bavures. A y regarder de plus près, la division fonctionnelle semble un peu courte. Rafles et politiques massives d'élimination se rencontrent dans les sociétés 
policièrement développées, quand le contexte de la violence l'exige - qu'on se souvienne des stades chiliens après le coup d'Etat de Pinochet - et concurremment la précision de la répression coexiste avec des massacres dans les zones où l'armée fait de la politique contre-insurrectionnelle. Le réseau familial, le lignage ethnique, les pratiques religieuses ou certaines solidarités économiques peuvent servir de fil discriminant pour une répression très fine, ce qui n'empêche pas, par ailleurs, que quelques règlements de compte personnels s'y ajoutent : psychologiques tant il est vrai que l'ennemi finit toujours par être détesté27, ou crapuleux. La "lisibilité" de la politique de disparition ne provient pas des spécificités de la répression ou de celle du quadrillage de la population, mais de leur inscription idéologique à l'intérieur d'un système répressif construit autour de la rhétorique de l'ennemi intérieur. La différence, car elle existe bien, ne se situe pas dans la panoplie de la violence, mais dans le cadre où elle s'insère. En revanche quand la figure de l'Etat se brouille, quand des féodalités, surtout régionales, se développent, quand la rudesse de la compétition réduit le gouvernement et ses agents à n'être plus qu'une faction parmi d'autres, les disparitions - et plus largement les violences - peuvent s'inscrire dans des calculs individuels ou dans des stratégies de groupes qui se substituent à l'enjeu national de la répression. En Colombie par exemple, les collusions entre militaires ou paramilitaires et trafiquants de drogue pour soustraire des terres à l'influence de la guérilla, transforment tous les protagonistes en simples éléments de réseaux locaux de pouvoir. La dynamique d'interaction qui s'établit à travers des rivalités, des concurrences, des retournements d'alliances n'est plus hiérarchisée à partir de l'acteur étatique (ou de ses représentants) mais banalisée par des pratiques de réciprocité entre acteurs équivalents. Cette privatisation de la violence sur fond d'indifférenciation accrue entre militaires et puissants régionaux, explique l'affaissement idéologique et l'instrumentalisation de disparitions ayant perdu toute lisibilité politique ${ }^{28}$.

3 L'espace ambigu des disparitions Nos analyses n'ont pas pu assigner les disparitions à un type particulier de violations des droits de l'homme - ce qui explique nos allers et retours entre disparitions et autres violences et par parenthèse, les difficultés méthodologiques pour isoler empiriquement la catégorie des disparus, puisque celle-ci est composée d'une suite de cas, ne faisant pas toujours sens en eux-mêmes ${ }^{29}$. Un tel rétrécissement idéologique, souligne bien sûr que le rapport politique l'emporte sur le simple rapport de violence, probablement parce que la légitimité s'inscrit dans un cycle plus long que celui de la violence, mais également à cause d'un choix qui privilégie le message destiné à la population sur celui dirigé vers adversaires. La rhétorique ami/ ennemi qui assurait l'impunité "stratégique" des forces de sécurité, n'est plus conçue que comme une parenthèse, une suspension provisoire de la démocratie ${ }^{30}$ dans l'espace - et dans le temps - particulier de la lutte contre la subversion. Sa légitimité est relativisée, encapsulée dans une histoire qui en considère la fin, et qui anticipe la modification des ressources politiques que cette fin entraînera pour les acteurs: aujourd'hui victime, demain adversaire, aujourd'hui traître tout juste bon à recevoir un châtiment, demain martyr réclamant des réparations et ayant de solides chances d'obtenir la condamnation de l'ex membre des forces de sécurité. La notion "d'information destructrice", qu'Erving Goffman ${ }^{31}$ utilise pour décrire les rôles contradictoires, peut, une fois transposée dans le domaine des disparitions, servir à conceptualiser les contraintes temporelles qui pèsent sur les conduites des acteurs. Une information destructrice est une information dont la circulation ruine, ou rend inutile, une représentation de la réalité. Elle produit un effet d'instabilité, entraîne un 
retournement brutal de situation qui explique les efforts de dissimulation consentis à son égard par ceux qui souhaitent maintenir valide "la définition de la situation" dit Goffman. Le paradoxe apparent que représente le refus d'assumer jusqu'au bout la violence exercée, alors même qu'elle paraît justifiée aux yeux du pouvoir, répond à un schéma analogue. Pour maîtriser le sens des événements politiques et éviter les effets de délégitimation, le pouvoir bloque "l'information destructrice" de la violence. Cette politique de secret peut concerner les informations destinées à la communauté internationale, ce qui est pour certains pays assez facile à réaliser. On a alors un contraste maximum entre l'ignorance au niveau international de la violence et sa connaissance interne. Le risque de délégitimation est fort à l'égard de la communauté internationale si l'information perce, mais ses effets seront nuls sur la scène intérieure déjà bien au fait des pratiques violentes. La rétention de l'information peut - cas le plus fréquent - concerner la population locale. Il s'agit alors de voiler certaines violences, d'en escamoter les traits les plus déplaisants par cette sorte de mise entre parenthèses que constitue une politique d'enlèvements et de disparitions. Son efficacité repose alors sur les complicités d'une population qui ne veut ni voir, ni savoir ${ }^{32}$. Cette conjoncture partisane réversible, non capitalisable n'est dans le fond que la dimension dynamique de l'espace que nous décrivions plus haut, où nous avons vu que les rapports de violence, les rapports politiques et les rapports de légitimité s'imbriquaient et s'entrechoquaient plus qu'ils ne se hiérarchisaient en une multitude de figures métonymiques. On ne s'étonnera pas dans ces conditions que la fluidité et la multiplicité de ces figures qui rendent compte de l'impact des disparitions sur tous les protagonistes, ne puissent être maîtrisées, et que face à cette contrainte la politique menée à propos des disparus renvoie pour les acteurs directs au coup tactique beaucoup plus qu'à la construction d'une stratégie.

4 Conclusion Tout au long de ce texte notre propos a été de décrypter le contexte qui sous-tend les pratiques des gouvernements soucieux de contrôler leur opposition politique. Pour cela après avoir décrit les disparitions comme complément endémique de la violence péri-guerrière, comme stratégie autonome de coercition, ou comme produit d'une logique d'interaction, nous avons souligné leur nature plus prédicative que substantive, et placé au premier plan dans l'analyse le jeu sur le sens qui leurs est consubstantiel. Cette dernière caractéristique qui confère aux disparitions une dimension symbolique très forte où, répétons-le, l'effet de sens prime sur les rapports proprement politiques, interdit à l'observateur une lecture classique qui en ferait un mode de régulation parmi bien d'autres des rapports pouvoir/opposition. En effet que cette lecture littérale soit cynique en montrant l'efficacité immédiate des enlèvements, ou critique en dénonçant les violations des droits de l'homme, elle méconnaît à chaque fois la dimension paradoxale d'une pratique dont rend bien compte la notion "d'information destructrice" que nous avons emprunté à E. Goffman. De ce point de vue, non seulement les disparitions nous renvoient à l'instabilité bien connue que provoque sur la mobilisation citoyenne l'usage continue de la violence politique (instrument efficace du pouvoir un jour, moyen de sa déstabilisation le lendemain), mais à travers l'analyse de la sorte de la capitalisation frauduleuse des ressources politiques qui les caractérise, elles dessinent une sorte de modèle qui nous invite à élargir l'espace ambigu où elles se meuvent à l'ensemble des pratiques irrégulières des pouvoirs. 


\section{NOTES}

1. L'acteur humanitaire interviendra s'il est doté d'une ressource politique lui permettant de peser efficacement sur le pouvoir. C'est le cas, à la suite d'un effet d'agrégation non prévu, pour les "folles de la place de mai" qui par leur obstination ont structuré autour du vide des disparitions la vie politique délégitimant le pouvoir des généraux argentins.

2. Voir sur ce thème le texte Didier Bigo, "Disparitions, coercition et violence symbolique".

3. Carl Schmitt La notion de politique, Champ Flammarion 1992. Pour la définition de la relation stratégique et du principe de la guerre voir Clausewitz, De la guerre, Les Editions de Minuit, 1955. Une application du concept ami/ennemi aux conflits dans le Tiers-Monde est donnée par Gilles Bataillon, "Contras et recontras nicaraguayens (1982-1993) : réflexions sur l'action armée et la constitution d'acteurs politicomilitaires", Cultures et Conflits, $n^{\circ} 12$, hiver 1993.

4. Discret : ce qui est voilé, mais également ce qui est séparé.

5. "Mode d'action sur les actions des autres" écrit Michel Foucault, Deux essais sur le pouvoir in Michel Foucault un parcours philosophique par Hubert Dreyfus et Paul Rabinau, Folio, 1992.

6. Mais c'est vrai de la plupart des violences contre les civils qui accompagnent les conflits ou les changements brutaux de régime : massacre anticommuniste à la prise de pouvoir de Suharto , massacre au Cambodge de Pol Pot, terreur rouge en Ethiopie avec l'arrivée de Mengistu. Citons en Asie la politique d'élimination discrète menée par les autorités indonésiennes au Timor-oriental, ou la répression des minorités nationales par les militaires birmans, ou les conflits des Etats successeurs de l'URSS, le Tadjikistan par exemple. En Afrique et en Amérique latine les massacres de civils dans les guerres civiles au Liberia, en Angola, au Mozambique, ou au Guatemala, au Salvador, en Colombie, au Pérou.

7. La guerre n'est pas l'action d'une force vive sur une masse morte, elle est toujours la collision de deux forces vives" Clausewitz, op. cit., Livre premier, §4.

8. Citons l'expression quasi-banalisée malgré sa dimension paradoxale "d'autogénocide cambodgien",voir également Génocide in Iraq : the Anfal campaign against the kurds. A Middle East Watch report, ou Timor, génocide oublié, Timor Information, Mars 1994. Voir Alfred Grosser, Le crime et la mémoire, Champ Flammarion, 1991. 9. Où rappelons le, la volonté de secret a abouti à l'occultation quasi-totale du processus d'extermination et à terme aurait empéché ou considérablement déformé son inscription dans la mémoire collective si la guerre avait tourné autrement. 10. Par exemple fuite des populations kurdes devant l'offensive de Saddam Hussein après l'échec du soulèvement du printemps 1991, politique de transmigration du gouvernement indonésien au Timor-oriental.

11. Cf. Ted Gurr et Barbara Harff, "Victims of the state : genocides, politicides \& group repression since 1945", in International Review of Victimology, 1989/1.

12. Voir sur ce thème Philipe Braud, "La violence politique : repères et problèmes", in La violence politique dans les démocraties européennes occidentales, l'Harmattan, Collection Cultures et Conflits, 1993.

13. Michel Foucault, op. cit., p 313. 
14. Responsables de $85 \%$ des violations des droits de l'homme au Pérou si on en croit un rapport d'Amnesty International

15. Voir plus loin "Dénombrer les disparitions" et la bibliographie citée par Francis Perrin.

16. Voir plus loin "Dénombrer les disparitions" et la bibliographie citée par Francis Perrin.

17. Voir plus loin "Dénombrer les disparitions" et la bibliographie citée par Francis Perrin.

18. Au Sri Lanka, LTTE :Liberation Tigers of Tamil Eealam, JVP Janata Vimukthi Peramuna, aux Philippines : NPA : New People's Army, branche armée du parti communiste philippin.

19. Selon des sources officielles colombiennes 1700 personnes ont été prises en otages par les différents mouvements de guerilla en Colombie de 1989 à 1991. L'objectif n'est pas de faire disparaître les gens mais d'obtenir une rançon ou de faire pression sur des responsables politiques et administratifs. Sur cette question voir plus loin l'article de Francis Perrin qui souligne combien la documentation est difficile à réunir bien que l'a priori idéologique qui a longtemps rendu le sujet tabou soit maintenant levé.

20. Gabriel Aguilera Peralta cité pour le Guatemala : La Main Blanche, La Nouvelle Organisation Anticommuniste, L'Organisation de l'Armée Secrète, Oeil pour Oeil, la Rose Pourpre.

21. Gabriel Aguilera Peralta cité pour le Guatemala : La Main Blanche, La Nouvelle Organisation Anticommuniste, L'Organisation de l'Armée Secrète, Oeil pour Oeil, la Rose Pourpre.

22. Voir le cas de Barek Touil, cité par Amnesty Inernational, disparu marocain marié à une américaine et pour lequel le Département d'Etat semble avoir été d'une remarquable passivité, persuadé que c'était la meilleure attitude à l'égard des autorités marocaines.

23. Exploitant à travers le relai américain, les analyses de la guerre révolutionnaire des militaires français en Algérie.

24. C'est au retour d'un stage aux USA que le colonel Contreras créa la D.I.N.A.

25. Le sort des prisonniers politiques était réglé soit par le chef de la D.D.S., parent d'Hissène Habré, soit directement par Hissène Habré, voir Amnesty International, "Tchad le cauchemar continu", avril 1993.

26. Général de division Khin Nyunt, premier secrétaire du SLORC, chef des services de renseignements de l'armée birmane, cité in "disparitions et assassinats", Amnesty International, mai 1991.

27. Clausewitz séparait avec soin l'intention hostile, qui seul fonde le rapport avec l'ennemi du sentiment d'hostilité qui en est la coloration psychologique occasionnelle. Les complots contre les chefs d'Etat dans les régimes de pouvoir personnel entrainent des répressions féroces où il est difficile de distinguer la dimension de calcul et la dimension de vengeance : Syrie, Irak, mais également Maroc où l'affaire Dlimi est à l'origine de nombreuses arrestations et disparitions qui montrent bien la dimension affective de la répression.

28. Voir l'article de D. Pécaut qui analyse en détail le phénomène de dépolitisation de la violence en Colombie. E. Meyer fait le même type de remarques pour le Sri Lanka.

29. Voir : Dénombrer les disparus.]. Nous n'avons pas non plus, réussi à les rattacher à un type unique de pratiques répressives d'Etat - éradication policière, terreur militaire - puisqu'elles participent à tous. Cela nous a obligé à réunir dans le même ensemble des 
pays comme l'Irak, le Cambodge des Khmers rouges, l'Indonésie des années 60, la Chine, tous pays de secret intérieur contrôlant fermement leurs frontières et peu réceptifs à la pression internationale, ce qui majore les risques de massacres, et ceux plus nombreux, plus ouverts, sensibles à leur image internationale, où les gouvernements sont obligés d'arbitrer entre l'élimination des adversaires internes et les effets désastreux que cela produit à l'extérieur. Et pourtant à l'évidence, le sens des disparitions n'y est pas identique. Dans les premiers pays, le terme disparition porte sur l'existence du conflit lui-même et produit de l'ignorance. Dans les seconds, sur sa gestion, il est lié au secret. Dans les premiers, disparition signifie escamotage et se rapporte à l'information sur le conflit plus qu'au détail des violences. Dans les seconds, se sont les effets de la répression qu'il faut faire disparaître, un peu à la manière des tortures dont les traces s'effacent en quelque jours. De ceci concluons que si le champ sémantique des disparitions ne correspond pas à un secteur autonome des politiques coercitives qu'on pourrait découper et décrire, et dont on pourrait même prévoir approximativement les effets en mettant en balance avantages et contraintes, c'est parce que les disparitions portent à la fois sur des événements et sur leur interprétation. Nous pouvons maintenant reprendre et approfondir la sociologie empirique des disparitions et de la coercition que nous avons esquissée plus haut. Définies uniquement comme rapport de violence, les disparitions sont des actions efficaces et nécessaires, qu'on peut dans le domaine technique différencier des autres violences. Elles renvoient à un surcroît de violence pour des victimes niées jusque dans leur existence et correspondent à des calculs tactiques visant à introduire par le secret, le doute chez l'adversaire. Ou, désidéologisées et instrumentalisées, elles se confondent très prosaïquement avec des commodités techniques de la répression : ni prison à gérer, ni traces administratives. Mais les disparitions sont aussi signes, messages. Elles ont une valeur de communication qu'il faut se garder de confondre avec leur dimension physique. Elles concourent à structurer la violence autour de la rhétorique ami/ennemi que nous avons évoquée à de nombreuses reprises, mais parallèlement elles dissimulent la violence aux yeux du plus grand nombre, favorisant par le silence l'élargissement du tiers indifférent ou complice. La lisibilité politique des disparitions devient alors paradoxale. Nous décrivions plus haut le grand nombre d'organisations paramilitaires et d'escadrons de la mort mêlés à cette violence. Cette floraison organisationnelle montre à l'évidence que la police et l'armée cherchent à dissimuler leurs responsabilités pour éviter des mises en cause directes. Ce refus d'assumer jusqu'au bout la violence exercée, alors même que cette dernière était justifiée dans le discours politique officiel ramène les disparitions au rang d'expédients provisoires et même honteux, dont la seule justification est l'urgence provoquée par une situation de crise[[Contestations sociale et politique au début des années 60 au Maroc, et lutte contre le Front Polisario quinze ans après, triomphe de l'unité populaire au Chili, développement de la guerilla urbaine en Argentine et en Urugay, tentatives séparatistes au Sri Lanka...Les défis qui menacent l'ordre social tout entier sont divers. 30. Sur ce thème voir Gilles Bataillon, "Réversibilité politique en Amérique latine", Passé Présent, $n^{\circ} 4$. L'auteur s'interroge sur la dimension transitoire des régimes militaires et suggère que l'emploi de la terreur ne renvoie pas forcément à un régime tyrannique, mais correspond plutôt à un modèle de suspension de la démocratie pour faire face à une situation d'urgence, dont un des modéles pourrait être la manière dont la France a géré la guerre d'Algérie. 
31. Erving Goffman, La mise en scène de la vie quotidienne, tome1, chapitre 4, Minuit 1973.

32. Erving Goffman, La mise en scène de la vie quotidienne, tome1, chapitre 4, Minuit 1973.

INDEX

Mots-clés : disparitions, invisible 